\title{
THE DISTANCE MAGIC INDEX OF A GRAPH
}

\author{
Aloysius Godinho, Tarkeshwar Singh \\ Department of Mathematics \\ Birla Institute of Technology and Science Pilani \\ K K Birla Goa Campus, NH-17B, Zuarinagar, Goa, India \\ e-mail: p2014001@goa.bits-pilani.ac.in \\ tksingh@goa.bits-pilani.ac.in \\ AND \\ S. Arumugam ${ }^{1}$ \\ National Centre for Advanced Research in Discrete Mathematics \\ Kalasalingam University Anand Nagar \\ Krishnankoil-626 126, Tamil Nadu, India \\ e-mail: s.arumugam.klu@gmail.com
}

\begin{abstract}
Let $G$ be a graph of order $n$ and let $S$ be a set of positive integers with $|S|=n$. Then $G$ is said to be $S$-magic if there exists a bijection $\phi: V(G) \rightarrow S$ satisfying $\sum_{x \in N(u)} \phi(x)=k$ (a constant) for every $u \in V(G)$. Let $\alpha(S)=\max \{s: s \in S\}$. Let $i(G)=\min \alpha(S)$, where the minimum is taken over all sets $S$ for which the graph $G$ admits an $S$-magic labeling. Then $i(G)-n$ is called the distance magic index of the graph $G$. In this paper we determine the distance magic index of trees and complete bipartite graphs.
\end{abstract}

Keywords: distance magic labeling, distance magic index, $S$-magic graph, $S$-magic labeling.

2010 Mathematics Subject Classification: $05 \mathrm{C} 78$.

\section{REFERENCES}

\footnotetext{
${ }^{1}$ Also at Department of Computer Science, Liverpool Hope University, Liverpool, UK; Department of Computer Science, Ball State University, USA.
} 
[1] S. Arumugam, D. Froncek and N. Kamatchi, Distance magic graphs-a survey, J. Indones. Math. Soc. Special Edition (2011) 11-26.

doi:10.22342/jims.0.0.15.11-26

[2] A. Godinho, T. Singh and S. Arumugam, On S-magic graphs, Electron. Notes Discrete Math. 48 (2015) 267-273.

doi:10.1016/j.endm.2015.05.040

[3] J.A. Gallian, A dynamic survey of graph labeling, Electron. J. Combin. (2014) \#DS6.

[4] M. Miller, C. Rodger and R. Simanjuntak, Distance magic labelings of graphs, Australas. J. Combin. 28 (2003) 305-315.

[5] A. Rosa, On certain valuations of the vertices of a graph, in: Theory of Graphs (International Symp., Rome, 1966), (Gordon and Breach, New York and Paris, 1967) 349-355.

[6] D.B. West, Introduction to Graph Theory (Prentice-Hall of India, 1999).

Received 28 June 2016

Revised 20 October 2016

Accepted 20 October 2016 